\title{
Characterization of the attP site of the integrative element PSAM2 from Streptomyces ambofaciens
}

\author{
Alain Raynal, Annick Friedmann, Karine Tuphile, Michel Guerineau \\ and Jean-Luc Pernodet
}

Author for correspondence: Alain Raynal. Tel: +331691562 10. Fax: +33169154544. e-mail: alain.raynal@igmors.u-psud.fr

Laboratoire de Biologie et Génétique Moléculaire, Institut de Génétique et Microbiologie, UMR CNRS 8621, Bât. 400, Université Paris-Sud, F-91405 Orsay Cedex, France

\begin{abstract}
PSAM2 is integrated into the Streptomyces ambofaciens chromosome through site-specific recombination between the element (attP) and the chromosomal (attB) site. The $43 \mathrm{kDa}$ integrase protein encoded by PSAM2 catalyses this recombination event. Tools have been developed to study site-specific recombination in Escherichia coli. In vivo studies showed that a 360 bp fragment of attP is required for efficient site-specific recombination and that int can be provided in trans. PSAM2 integrase was purified and overexpressed in $E$. coli and Int binding at the attP site was studied. DNasel footprinting revealed two sites that bind integrase strongly and appear to be symmetrical with regard to the core site. These two $\mathrm{P1} / \mathrm{P2}$ arm-type sites both contain a 17 bp motif that is identical except at one position, GTCACGCAG(A/T)TAGACAC. $P 1$ and P2 are essential for site-specific recombination.
\end{abstract}

Keywords: actinomycetes, integrase, site-specific recombination, attachment site

\section{INTRODUCTION}

As well as their complex life cycle and industrial importance, Streptomyces spp. are also remarkable due to the accessory genetic elements they harbour. Integrative elements, which have only been found in actinobacteria, are present as integrated chromosomal copies. During mating with a strain lacking them, these integrative elements are able to excise, to transfer and to integrate at a specific site in the chromosome of their new host. Most integrative elements have similar functions to plasmids, such as pock formation associated with transfer, and are able to replicate autonomously. Such elements have been isolated from various actinobacteria belonging to the genera Streptomyces, Saccharopolyspora, Micromonospora and Amycolatopsis. The integration and excision processes have been studied in some of these elements [pSAM2 (Boccard et al., 1988), SLP1 (Omer \& Cohen, 1986), pMEA100 (Madon et al., 1987), pSG1 (Cohen et al., 1985), pIJ408 (Sosio et al., 1989), pSE211 (Brown et al., 1990), pSE101 (Brown et al., 1994) and pMEA300 (Vrijbloed et al., 1994)] and shown to involve site-specific recombination.

pSAM2 is an $11 \mathrm{~kb}$ element, originally isolated from Streptomyces ambofaciens, which produces the macrolide antibiotic spiramycin (Pernodet et al., 1984). pSAM2 can replicate, is self-transmissible, elicits the lethal zygosis reaction (pock formation) and mobilizes chromosomal markers (Smokvina et al., 1991). Furthermore, pSAM2 has a recombination system that is very similar to that of temperate phages. pSAM2 is integrated into the chromosome through site-specific recombination between the element $(a t t P)$ and the chromosomal (attB) sites. These regions share a $58 \mathrm{bp}$ segment extending from the anticodon loop to the $3^{\prime}$ end of a tRNA ${ }^{\text {Pro }}$ gene (Mazodier et al., 1990; Boccard et al., 1989).

In a previous study (Raynal et al., 1998), the pSAM2 sitespecific recombination system was reconstructed in Escherichia coli and the chromosomal attB site was characterized by use of two compatible plasmids, one carrying the attP site and expressing the pSAM2 integrase and the other carrying various fragments of the chromosmal attB region. With this system, it was shown that Int is the only Streptomyces protein required for site-specific integration and that Int and Xis are both required for site-specific excision. The attB region required for efficient site-specific recombination was shown to be $26 \mathrm{bp}$ long and centred around the anticodon loop. Comparison of this $26 \mathrm{bp}$ region with attP suggested, according to the lambda model (Campbell, 1992), that B and B', and C and C', core-type Int binding sites consist of $9 \mathrm{bp}$ imperfect inverted repeats separated by a 5 bp overlap region. 
This study aimed to characterize the attP site with respect to its minimal size and to the integrase binding sites.

\section{METHODS}

Strains, plasmids and media. E. coli DH5 $\alpha$ (Hanahan, 1983) was used for cloning experiments and plasmid DNA propagation. pCRII (Invitrogen) was used for cloning PCR products, and pBluescript (Stratagene) was used for cloning experiments. pMCL200, a low-copy-number p15A derivative (Nakano et al., 1995), was used for the construction of attPcontaining plasmids. Integrase was overexpressed by use of a pET-21a $(+)$ vector in E. coli BL21(DE3) (Novagen). The attB-containing plasmids, pOSattB and pOSint $1 \Delta \mathrm{P}$, a pOSint 1 derivative expressing int (Raynal et al., 1998) and deleted for the attP site (see Fig. 1), were used for in vivo recombination experiments.

Bacteria containing plasmids were routinely grown on LB agar supplemented with $100 \mu \mathrm{g}$ ampicillin (Ap) $\mathrm{ml}^{-1}, 40 \mu \mathrm{g}$ streptomycin $(\mathrm{Sm}) \mathrm{ml}^{-1}, 20 \mu \mathrm{g}$ chloramphenicol $(\mathrm{Cm}) \mathrm{ml}^{-1}$. Liquid $\mathrm{LB}$ was supplemented with $50 \mu \mathrm{g} \mathrm{Ap} \mathrm{ml}^{-1}, 20 \mu \mathrm{g} \mathrm{Sm} \mathrm{ml}{ }^{-1}, 10 \mu \mathrm{g}$ $\mathrm{Cm} \mathrm{ml}^{-1}$.

Construction of attP-containing plasmids. The complete attP region was isolated from pTS33 (Smokvina et al., 1990) as an

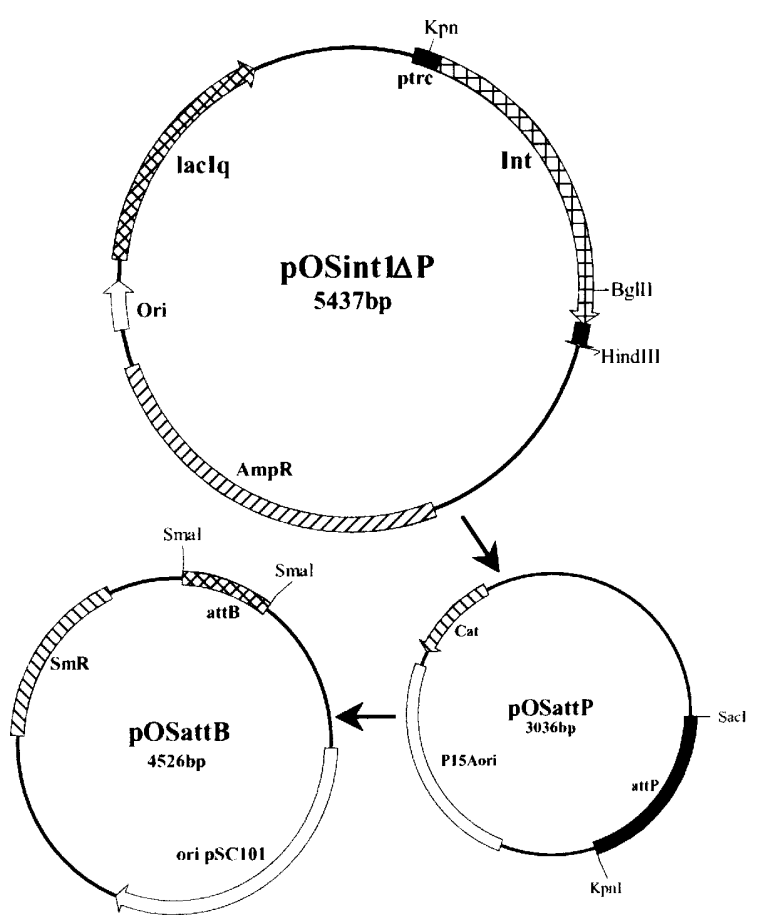

Fig. 1. Structures of attP- and attB-containing plasmids. pOSint $1 \Delta \mathrm{P}$ is an integrase-producing plasmid deleted for the attP site; replicative at $37{ }^{\circ} \mathrm{C}, A p^{\mathrm{R}}$. pOSattP shows a schematic structure of all the attP-containing plasmids. Replicative at $37^{\circ} \mathrm{C}, \mathrm{Cm}^{\mathrm{R}}$. pOSattB is a plasmid carrying a $400 \mathrm{bp}$ Smal-Smal fragment including the chromosomal attB site; replicative at $30{ }^{\circ} \mathrm{C}, \mathrm{Sm}^{\mathrm{R}}$. As a first step attP-containing plasmids were introduced into the strain expressing integrase. As a second step, an attB-containing plasmid was introduced into the resulting strain in order to select $\mathrm{Sm}^{\mathrm{R}}$ colonies at $42^{\circ} \mathrm{C}$.
AvrII-BglII fragment whose AvrII extremity was blunt-ended by Klenow treatment. The resulting fragment was cloned into EcoRV/BamHI-digested pMCL200 vector generating pOSattPpSAM2. The minimal attP site and parts of this site $(\mathrm{a}, \mathrm{b}$, c) (Fig. 2) were cloned either by subcloning or by PCR amplification. pOSattPbc was derived from pOSattPpSAM2 after XbaI-DraIII deletion in the $5^{\prime}$ region followed by a SalI-SalI deletion in the $3^{\prime}$ region. Deletion was performed by generating blunt ends with Klenow treatment, followed by ligation.

attPmin, attPab and $a t t P b$ were generated by PCR amplification using the PminX/PminE, PminX/PB3 and PB5/PB3 primer pairs, respectively (Fig. 2). (Oligonucleotides: PminX, 5'-GGACGGCATCCTCGAGCGGGGTCC-3'; PminE, 5'CGCGAATTCGTCGACGAGCAGGCCGGTGAGTG3'; PB5, 5'-GCGATCGATCTCACCTGGTGTTTCTCTGTC-3' ; PB3, 5'-CGCGAATTCCACTGGCCCAAGGTCGAGC-3' ${ }^{\prime}$; restriction sites present in the primers are underlined.) The amplified fragments were cloned into pMCL200 as XhoI-EcoRI, XhoI-EcoRI and ClaI-EcoRI fragments, respectively. PCR was performed on a TECHNE thermocycler (Fisher) using Taq DNA polymerase (Amersham Pharmacia Biotech). Synthetic oligonucleotides were provided by Genosys Biotechnologies.

Construction of the integrase expression plasmid. To overproduce and to purify the pSAM2 integrase, the int gene was placed under the control of the T7 promoter of the E. coli plasmid pET-21a $(+)$ (Novagen) in which the basal expression is very low in the absence of IPTG induction. The int gene was PCR amplified from pOSint1 (Raynal et al., 1998) with the Int5B/IntTag primer pair. A BamHI site was introduced immediately upstream of the int ATG start codon by use of the Int5B (5'-TTTGGATCCATGGCCAAGCGACGTAGC-3') synthetic sequence.

The IntTag sequence (5'-GTGCTCGAGTCGCGCCGGTCCCCGCTTG-3') hybridizes to the $3^{\prime}$ end of the integrase coding sequence. The cloning of a $B a m \mathrm{HI}-\mathrm{Xhol}$ amplified fragment in $\mathrm{pET}-21 \mathrm{a}(+)$ led to an in-frame fusion, providing a His-Tag coding sequence at the C-terminus of the integrase. pET-21int was obtained in DH5 $\alpha$ and then introduced into BL21(DE3) to overproduce integrase after IPTG induction.

Purification of integrase. The int gene was cloned in the expression vector, pET21a +, under the control of the IPTGregulated T7 promoter, yielding pET21-int. A His-tag was added to the $\mathrm{C}$-terminal end of the integrase. Five millilitres of a $37^{\circ} \mathrm{C}$ overnight preculture of E. coli BL21(DE3) containing pET-21int were inoculated into $500 \mathrm{ml} \mathrm{LB}$ medium containing $100 \mu \mathrm{g}$ ampicillin $\mathrm{ml}^{-1}$. The culture was grown at $22^{\circ} \mathrm{C}$ with shaking to an $\mathrm{OD}_{650}$ of 0.45 . IPTG was then added $(1.0 \mathrm{mM})$ and the cells were grown for a further $5 \mathrm{~h}$ at the same temperature and harvested by centrifugation. After washing once in $50 \mathrm{mM}$ Tris, $100 \mathrm{mM} \mathrm{NaCl}, \mathrm{pH} \mathrm{8}$, the pellet was resuspended in $5 \mathrm{ml}$ of the same buffer and frozen at $-20^{\circ} \mathrm{C}$ before lysis. Lysozyme $\left(1 \mathrm{mg} \mathrm{ml}{ }^{-1}\right)$ was added and the sample was lysed for $30 \mathrm{~min}$ at room temperature. After centrifugation the pellet was resuspended in the same buffer containing $0 \cdot 1 \%$ sodium deoxycholate. The resulting suspension was denatured by sonication and centrifuged at $13000 \mathrm{~g}$ for $10 \mathrm{~min}$. The resulting supernatant was further purified, as described by Novagen, and the soluble fraction was found to contain only a small amount of integrase. Therefore, integrase was purified from inclusion bodies present in the pellet following the last centrifugation step. The pellet was resuspended in binding buffer $(5 \mathrm{mM}$ imidazole, 


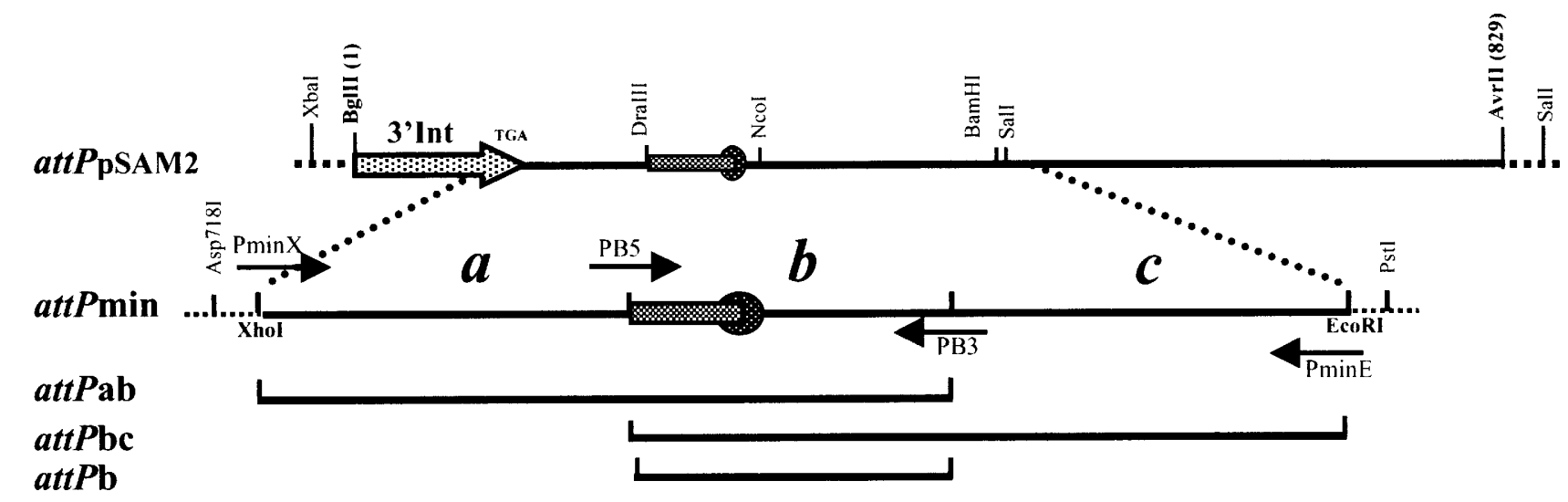

Fig. 2. Schematic representation of the attP site located immediately downstream of the Int coding sequence. The Avrll-Bg/ll fragment was isolated from pSAM2. All the restriction sites used in subcloning experiments are represented. attPpSAM2, attPmin, attPab, attPbc and attPb were cloned in pMCL200. attPmin was cloned in pBluescript KS+ for footprint experiments. The shaded bars represent the $58 \mathrm{bp}$ identity segment; the circles at the end of the shaded bars (core site) show the localization of the recombination point; the black arrows indicate the position and orientation of the oligonucleotides used for PCR amplification.

$0 \cdot 5 \mathrm{M} \mathrm{NaCl}, 20 \mathrm{mM}$ Tris/ $\mathrm{HCl} \mathrm{pH} 7 \cdot 9$ ) containing $6 \mathrm{M}$ urea (final concentration). All further purification steps (loading, washing and elution) were performed with buffer containing $6 \mathrm{M}$ urea. Proteins were eluted in $300 \mathrm{mM}$ imidazole, $0.5 \mathrm{M}$

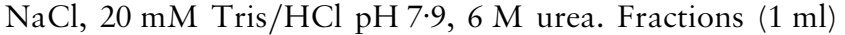
were eluted from the His-bind resin column, and the $A_{595}$ revealed that integrase was in fractions 2, 3 and 4. Urea was gradually removed by successive dialysis to a final urea concentration of $1.5 \mathrm{M}$ to allow protein refolding. A $20 \%$ $(\mathrm{v} / \mathrm{v})$ glycerol solution containing $0.6 \mathrm{mg}$ integrase $\mathrm{ml}^{-1}$ was split into aliquots and stored at $-20^{\circ} \mathrm{C}$. SDS-PAGE was carried out in $10 \%$ acrylamide gels according to Sambrook et al. (1989). Proteins were detected by Coomassie blue staining.

DNase I footprinting. DNase I footprinting experiments were performed by use of the Promega Core Footprinting System. The probes consisted of fragments carrying a $3^{\prime}$ and a $5^{\prime}$ protruding end isolated from the cloned attP minimal site. The $5^{\prime}$ end was radio-labelled by filling in with the Klenow fragment of DNA polymerase I and $\left[\alpha-{ }^{32} \mathrm{P}\right]$ CTP. The labelled fragment was loaded onto an agarose gel and purified by use of the GFX Gel Band Purification Kit (Amersham Pharmacia Biotech). Binding reactions were carried out in $25 \mathrm{mM}$ Tris/HCl pH 8.0, 6.25 mM MgCl, $0.5 \mathrm{mM}$ EDTA, $0.5 \mathrm{mM}$ DTT, $200 \mathrm{mM} \mathrm{KCl}$ and $10 \%$ (v/v) glycerol buffer. Purified integrase $(10 \mu \mathrm{g})$ was incubated with 50000 c.p.m. of labelled probe at $25^{\circ} \mathrm{C}$ for $1 \mathrm{~h}$. Reactions were treated with $1.5 \mathrm{U}$ DNase I (RQ1 DNase Promega) for $1 \mathrm{~min}$ at $25^{\circ} \mathrm{C}$ and the resulting product was analysed on a $6 \%$ sequencing gel.

\section{RESULTS}

\section{Tripartite system used for the study of attP in E. coli}

pSAM2 site-specific recombination is functional in $E$. coli and requires the attP and $a t t B$ sites and integrase production (Raynal et al., 1998). In these experiments, the three partners were carried by three different compatible plasmids. int, carried by pOSint $\Delta \mathrm{P}$, was expressed under the control of the IPTG-inducible ptrc promoter on an $\mathrm{Ap}^{\mathrm{R}}$ multicopy plasmid, derived from
pTrc99A (Amann et al., 1988) and replicating with a ColE1 replication origin. The attB site was carried by pOSattB, as previously described (Raynal et al., 1998). This low-copy-number plasmid, constructed from pGB2ts (Clerget, 1991), a pSC101 derivative (Churchward, 1984), confers $\mathrm{Sm}^{\mathrm{R}}$ and replicates at $30^{\circ} \mathrm{C}$ but not at $42{ }^{\circ} \mathrm{C}$. The pSAM2 attP site was carried by pMCL200, a low-copy-number p15A derivative (Nakano et al., 1995), conferring $\mathrm{Cm}^{\mathrm{R}}$. The attP and $a t t B$ sites were carried by vectors sharing no sequence identity. To test the functionality of the pSAM2 site-specific recombination system, we aimed to select co-integrates arising from site-specific recombination between attB and att $P$.

An E. coli $\mathrm{DH} 5 \alpha$ strain harbouring pOSint $\Delta \mathrm{P}$ for the production of pSAM2 integrase was first transformed by pOSattP. The resulting clones were selected as $A^{\mathrm{R}} /$ $\mathrm{Cm}^{\mathrm{R}}$ colonies at $37^{\circ} \mathrm{C}$. The plasmids used are stably maintained in E. coli, at least in the presence of a selection pressure, and before transformation with the third plasmid, recipient cells retained the two other plasmids. These strains were further transformed by pOSattB. After $4 \mathrm{~h}$ expression in the presence of IPTG $\left(5 \times 10^{-4} \mathrm{M}\right)$ to allow expression of the $\mathrm{Sm}^{\mathrm{R}}$ phenotype, incubation was continued overnight at $30^{\circ} \mathrm{C}$ in the presence of both $\mathrm{Sm}\left(20 \mu \mathrm{g} \mathrm{ml}^{-1}\right)$ and IPTG. Finally, $\mathrm{Sm}^{\mathrm{R}}$ clones were selected at $30^{\circ} \mathrm{C}$ and $42^{\circ} \mathrm{C}$, on plates containing $\mathrm{Sm}\left(40 \mu \mathrm{g} \mathrm{ml}^{-1}\right)$ and IPTG. As pOSattB, conferring $\mathrm{Sm}^{\mathrm{R}}$, cannot replicate at $42{ }^{\circ} \mathrm{C}$, the growth of $\mathrm{Sm}^{\mathrm{R}}$ clones at $42^{\circ} \mathrm{C}$ should indicate the formation of pOSattB-pOSattP co-integrates by site-specific recombination. $\mathrm{Sm}^{\mathrm{R}}$ colonies obtained at $42^{\circ} \mathrm{C}$ were replica plated to test for $\mathrm{Cm}^{\mathrm{R}}$ at $42{ }^{\circ} \mathrm{C}$. All the tested $\mathrm{Sm}^{\mathrm{R}}$ colonies isolated at $42{ }^{\circ} \mathrm{C}$ were $\mathrm{Cm}^{\mathrm{R}}$. In all cases, restriction analysis on plasmids isolated from these strains confirmed that they were co-integrates resulting from recombination between $a t t B$ and attP, even when the recombination efficiency was very low. In the 
Table 1. Recombination between attP and attB on plasmids in $E$. coli

All the plasmids containing the 'native' attP site and its derivatives are presented, with the origin of the inserts (subcloning or PCR amplification) and their sizes. The estimated recombination efficiencies between pOSattP and pOSattB plasmids are given as percentages. These values are means obtained with three independent experiments. In each experiment, $100 \%$ represented more than 1500 colonies.

\begin{tabular}{|llccc|}
\hline $\begin{array}{l}\text { attP-containing } \\
\text { plasmid }\end{array}$ & Cloned fragment & Insert origin & Insert size (bp) & $\begin{array}{c}\text { Recombination } \\
(\%)\end{array}$ \\
\hline pOSattPpSAM2 & BglII-AveII & Subcloned & 829 & 94 \\
pOSattPmin & XhoI-EcoRI & PCR & 361 & 92 \\
pOSattPbc & DraIII-SalI & Subcloned & 269 & $0 \cdot 37$ \\
pOSattPab & XhoI-EcoRI & PCR & 195 & $0 \cdot 19$ \\
pOSattPb & ClaI-EcoRI & PCR & 115 & $2 \cdot 5$ \\
\hline
\end{tabular}

absence of the int gene, no $\mathrm{Sm}^{\mathrm{R}}$ colonies were obtained at $42{ }^{\circ} \mathrm{C}$. The co-integrates were therefore due to sitespecific recombination promoted by Int.

As $\mathrm{Cm}^{\mathrm{R}} \mathrm{Sm}^{\mathrm{R}}$ colonies growing at $42^{\circ} \mathrm{C}$ were only obtained if co-integrates were formed, the ratio of $\mathrm{Sm}^{\mathrm{R}}$ colonies isolated in non-permissive $\left(42{ }^{\circ} \mathrm{C}\right)$ and permissive $\left(30^{\circ} \mathrm{C}\right)$ conditions for pOSattB replication allowed us to measure the relative frequency of recombination. With pOSattPpSAM2, harbouring a $829 \mathrm{bp}$ fragment containing the attP region, this ratio was $94 \%$, confirming that pSAM2 site-specific recombination is very efficient in E. coli (Table 1) even when Int acts in trans on attP sites. Intermolecular recombination between $a t t P$ and $a t t B$ was assayed in our experiments and the conclusions reached about the sequences required do not necessarily apply for the excision reaction.

\section{Identification of the minimal attP functional site}

Given the proposed similarity between the organization of the pSAM2 attP and the attP sites of other temperate phages (Boccard et al., 1989), we expected to find armtype Int binding sites on both sides of the core-type Int binding site. Therefore we amplified, as a putative minimal attP site, a fragment extending about $200 \mathrm{bp}$ either side of the attP core region (Fig. 2). This $360 \mathrm{bp}$ fragment (attPmin) was tested for in vivo site-specific recombination in the same way as attPpSAM2 and gave $92 \%$ of co-integrates. Thus, this fragment contains all the sites required for efficient recombination. This attPmin site was further divided into three regions, $a, b$ and $c$ : $b$ containing the core binding site, $a$ and $c$ containing the putative arm-type sites (Fig. 2). Various fragments corresponding to regions $\mathrm{a}$ and $\mathrm{b}$ (attPab), b alone $(a t t P b)$ and $b$ and $c(a t t P b c)$ were tested for their ability to recombine. Although att $\mathrm{Pb}$ contained the 58 bp identity segment, shared by att $P$ and $a t t B$, and the core site where recombination takes place, it did not recombine efficiently, demonstrating that sequences present in a and/or c are required. The results obtained with attPab and attPbc (Table 1) clearly showed that the a and $\mathrm{c}$ regions are involved in the recombination event. The attPmin site was further analysed for in vitro binding of the integrase.

\section{Purification of integrase}

Before purifying integrase, the functionality of the Histagged integrase was studied in vivo. As for the study of the attP site, we used pOSattPmin and pOSattB to provide recombinant sites and pET21-int to produce the His-tagged integrase in recombination experiments performed in the expression strain BL21(DE3). The frequency of recombination was $85 \%$ with pET21-int, compared to $92 \%$ with pOSint $1 \Delta \mathrm{P}$ in $\mathrm{DH} 5 \alpha$. These results clearly show that the His-tagged integrase encoded by pET21-int was functional and bound efficiently to its target sequences.

Integrase was purified from inclusion bodies using Hisbind resin. After the purification of these inclusion bodies, integrase was recovered under denaturing conditions (6 M urea) followed by partial renaturation. Approximately $2 \mathrm{mg}$ purified integrase was obtained from $500 \mathrm{ml}$ culture. Analysis of the final product by $10 \%$ SDS-PAGE showed a unique band at $43 \mathrm{kDa}$, corresponding to the purified integrase (Fig. 3).

\section{Study of Int binding sites in attP by footprinting analysis}

To determine the precise location of the Int binding sites, we investigated the ability of Int to protect attP against DNaseI cleavage. Firstly, the XhoI-EcoRI fragment corresponding to the attPmin region was cloned into pBluescript KS+. The Asp718I-DraIII (104 bp) and NcoI-PstI (165 bp) fragments were isolated from the resulting plasmid and labelled at their $5^{\prime}$ protruding end, respectively Asp718I and NcoI. Asp718I and PstI sites were provided by the multiple cloning site of the vector. After purification on agarose gel, the resulting labelled fragments were used to analyse binding on the top strand of the a and c regions (Fig. 2). In the same way, the BamHI-DraIII fragment (226 bp) labelled at 


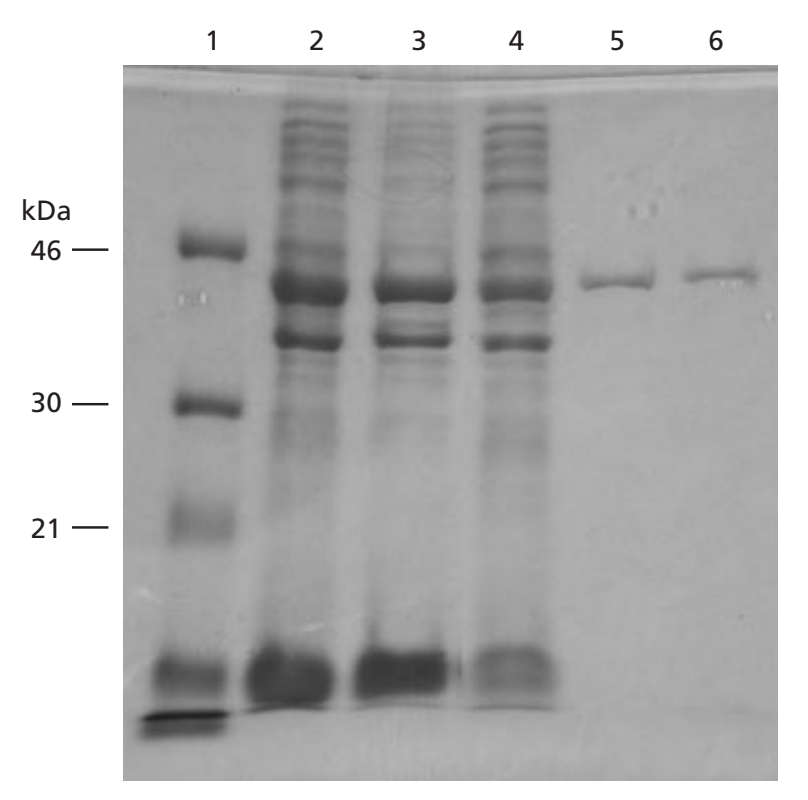

Fig. 3. Purification of overexpressed His-tagged Int from E. coli. The fractions from each purification step were analysed on $10 \%$ SDS-PAGE and Coomassie blue stained. Lanes: 1, Molecular mass markers; 2, total $E$. coli crude extract; 3, purified inclusion bodies after $0.45 \mu \mathrm{m}$ filtration; 4 , soluble fraction; 5/6, fractions 2 and 3 eluted from the His-bind column.

the BamHI end was used to analyse the protected sequence on the bottom strand of the $\mathrm{c}$ region of the attP site. After integrase binding and DNaseI digestion, the final products were analysed on a $6 \%$ sequencing gel. The results showed two domains of $28 \mathrm{nt}$ protected from the DNaseI digestion, located in the a and c region of the attP site (Fig. 4). These domains show the same protection patterns: strongly protected $6 \mathrm{nt}, 9 \mathrm{nt}$ and $7 \mathrm{nt}$ motifs separated by two blocks of three weakly or not protected nucleotides. This weak protection at certain positions may be due to the use of a high $\mathrm{K}^{+}$ concentration $(200 \mathrm{mM})$, which increases specificity but decreases the affinity of protein-DNA binding.

In accordance with the nomenclature used in the lambda system, these two protected domains were designated as arm-type sites. Comparison of the two arm-type sites in the protected regions revealed a common $17 \mathrm{nt}$ sequence with a single mismatch: GTCACGCAG(A/ T)TAGACAC. This sequence is probably the target of Int. This sequence was absent in the central (b) region of attPmin and was only found twice in the complete attPmin region. These two arm-type sites were called P1 and P2. Several attempts have been made to investigate the putative binding of integrase at or around the core site, but no binding was observed in our experimental conditions, on a fragment carrying the core site alone or both the core site and the P1 defined site, even in low $\mathrm{K}^{+}$ concentration $(50 \mathrm{mM})$, which increases the binding affinity. This observation was not really surprising and confirmed that although the core binding site is essential for recombination, it binds Int more weakly than the arm sites (Dorgai et al., 1998; Tirumalai et al., 1998).
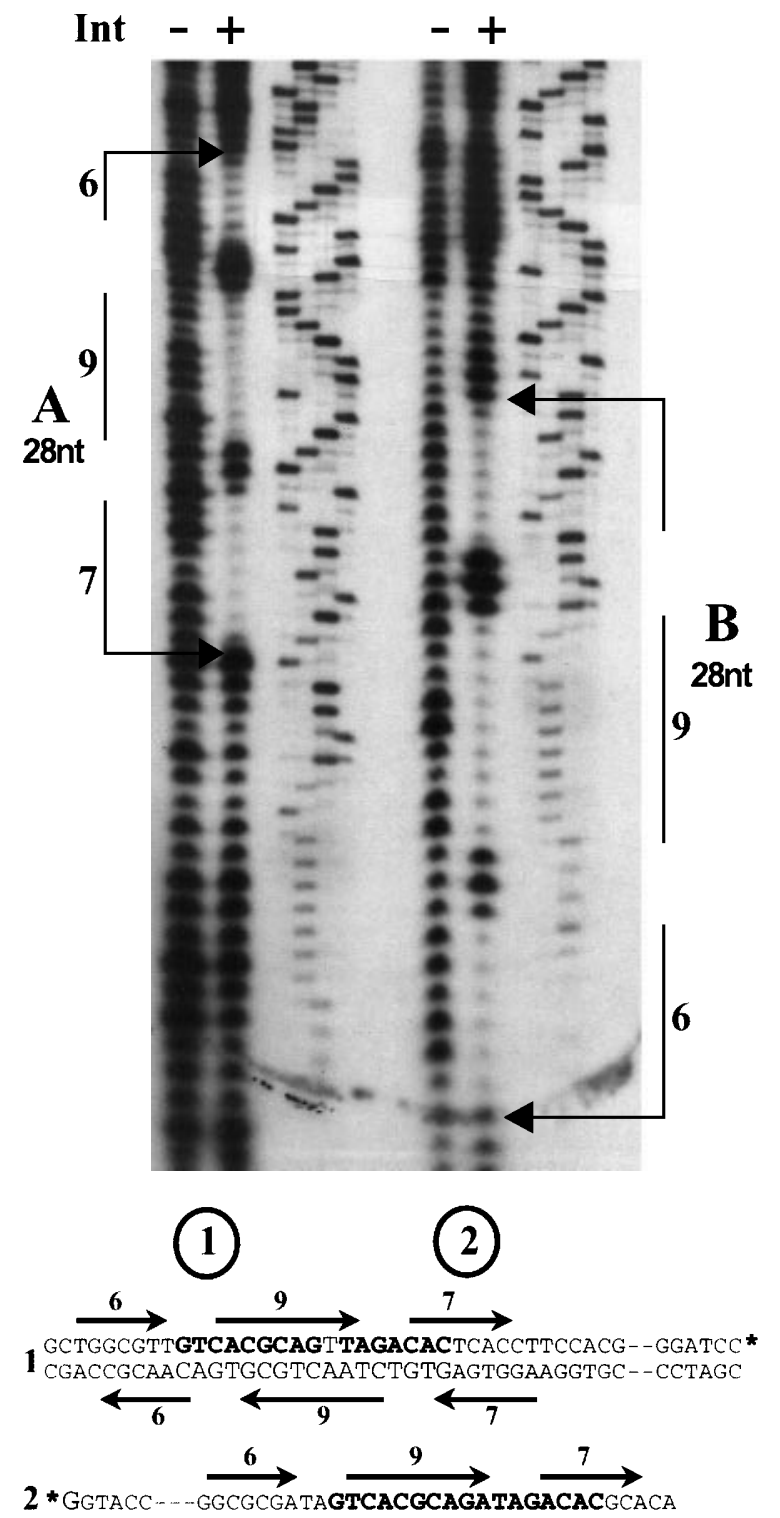

\section{GTCACGCAGA/TTAGACAC}

Fig. 4. DNasel footprinting of attP-Int complexes. 1, DNasel footprinting of the complex between Int and attP DNA 5' labelled (BamHI site) at the $3^{\prime}$ end of the attP site. Only the bottom strand is presented. 2, DNasel footprinting of the complex between Int and attP DNA 5' labelled (Asp718l site) at the $5^{\prime}$ end of the attP site. The top strand is presented. - and + indicate respectively absence or presence $(10 \mu \mathrm{g})$ of integrase. Due to the presence of compression in the sequencing gel of the attP region, because of $21 \mathrm{bp}$ GC-rich inverted repeats, the size marker presented here is the pBluescript KS + vector sequenced starting from the T3 universal primer. The $17 \mathrm{nt}$ repeat (16/17) sequence found in both the $A$ and $B$ regions is boxed; these repeat sequences are considered as arm-type sites called P1 and P2 (see Fig. 5).

If we represent the pSAM2 minimal attP site with a coordinate 0 at the centre of our previously described core site, the $17 \mathrm{bp}$ repeat sequences lie between 


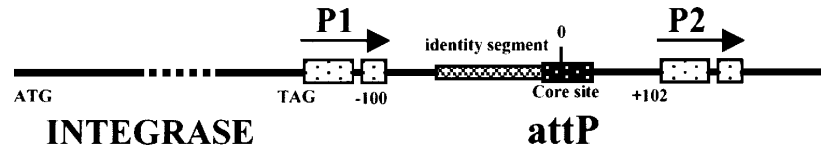

Fig. 5. Schematic representation of the entire region including both the Int coding sequence and the attP site. P1 and P2 armtype sites are indicated.

positions $-100 /-116$ in the $5^{\prime}$ region and $+102 /+118$ in the $3^{\prime}$ region, represented as $\mathrm{P} 1$ and P2 in Fig. 5. Thus, the two defined arm sites, which strongly bind integrase, appear to be symmetrical with regard to the core site at which the recombination event occurs.

\section{DISCUSSION}

In this work we have shown that a 360 bp region of the pSAM2 attP site is required for efficient recombination between $a t t B$ and $a t t P$. This region contains the coretype site and two arm-type-binding sites, P1/P2, for the Int protein.

As pSAM2 site-specific recombination is efficient in E. coli (Raynal et al., 1998), no Streptomyces-specific protein, besides Int, and Int together with Xis for excision, seems to be required for this process. However, the involvement of some E. coli proteins, e.g. histonelike proteins, cannot be excluded.

pSAM2 integrase resembles other integrases in its Cterminal part (Nunes-Düby et al., 1998) and this part is most probably involved in the catalytic activity. For the $\mathrm{N}$-terminal part of pSAM2 Int, possibly involved in arm-binding activity, no obvious conserved region was detected after comparison with other integrases. pSAM2 integrase appears to have a strong affinity for arm-type sites, but no integrase binding could be detected on the core-type site, even in low $\mathrm{KCl}$ concentrations, which decrease binding specificity. It seems that the binding of Int to arm-type sites is a prerequisite for proper recognition of the core binding site during integration.

The overall organization of the pSAM2 attP site can be compared to those of other phages such as P22, P2, lambda and L5 (Pena et al., 1997) (Fig. 6). In all these cases, the arm-type sites are located between $-60 /-130$ and $+60 /+120 \mathrm{nt}$ on both sides of the core site. For pSAM2, only two arm-type sites, consisting of $17 \mathrm{nt}$ direct repeat sequences with a single mismatch, are required. In lambda (Ross \& Landy, 1982), P2 (Yu \& Haggard-Ljungquist, 1993), P22 (Smith-Mungo et al., 1994) and mycobacteriophage L5 (Pena et al., 1997), the sequences of the arm-type sites are shorter (about $10 \mathrm{nt}$ ). For lambda and L5 phages, arm-type binding sites consist of four or five repeats of shorter sequences, but some of them do not appear to be essential, for example P'1/P2 for lambda (Bauer et al., 1986), P3/P6-7 for L5 (Pena et al., 1997). There is little or no sequence similarity between the arm-type sites of these four phages. Nevertheless, the site we described for pSAM2 shares five nucleotides with the lambda consensus (GTCAC). In the five arm-type binding sites described for lambda, the nucleotides TCA are perfectly conserved in the consensus sequence. In addition, arm-type mutants that contain a C-to-T transition, located in the lambda $\mathrm{P} 1, \mathrm{P}^{\prime} 2$ or $\mathrm{P}^{\prime} 3$ arm-type site, exhibit a 10- to 100 fold reduction in integrative recombination (Bauer et al., 1986). In contrast to phage lambda, for L5, P2 and P22, arm-type sites are arranged in pairs and it was suggested for L 5 that Int might bind co-operatively to pairs of sites. However, recent results (Pena et al., 1999, 2000) clearly indicate that the formation of site-specific recombination complexes in L5 is quite different to that in lambda.

The structural organization of the pSAM2 attP appears to be simpler than that of the other attP sites studied to

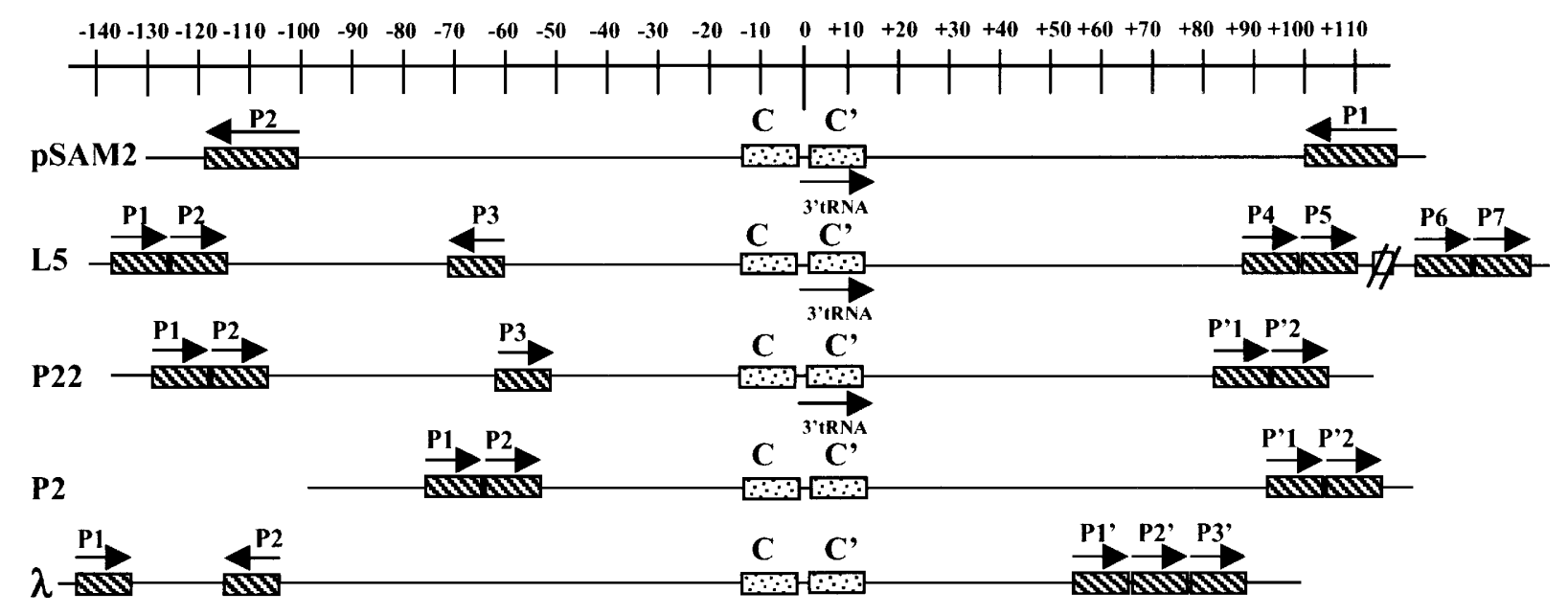

Fig. 6. Comparison of attP sites. The structures of the attP sites of pSAM2, L5, P22, P2 and lambda are presented, centred on the aligned core sites. The positions of arm-type and core-type integrase binding sites are shown and their relative orientation indicated by an arrow. 
date. It only contains two arm-type binding sites, but they are longer and are both essential for recombination.

\section{REFERENCES}

Amann, E., Ochs, B. \& Abel, K. J. (1988). Tightly regulated tac promoter vectors useful for the expression of unfused and fused proteins in Escherichia coli. Gene 69, 301-315.

Bauer, C. E., Hesse, S. D., Gumport, R. I. \& Gardner, J. F. (1986). Mutational analysis of integrase arm-type binding sites of bacteriophage lambda. Integration and excision involve distinct interactions of integrase with arm-type sites. J Mol Biol 192, 513-527.

Boccard, F., Pernodet, J. L., Friedmann, A. \& Guerineau, M. (1988). Site-specific integration of plasmid pSAM2 in Streptomyces lividans and S. ambofaciens. Mol Gen Genet 212, 432-439.

Boccard, F., Smokvina, T., Pernodet, J. L., Friedmann, A. \& Guerineau, M. (1989). The integrated conjugative plasmid pSAM2 of Streptomyces ambofaciens is related to temperate bacteriophages. EMBO J 8, 973-980.

Brown, D. P., Idler, K. B. \& Katz, L. (1990). Characterization of the genetic elements required for site-specific integration of plasmid pSE211 in Saccharopolyspora erythraea. J Bacteriol 172, 18771888.

Brown, D. P., Idler, K. B., Backer, D. M., Donadio, S. \& Katz, L. (1994). Characterization of the genes and attachment sites for site-specific integration of plasmid pSE101 in Saccharopolyspora erythraea and Streptomyces lividans. Mol Gen Genet 242, 185-193.

Campbell, A. M. (1992). Chromosomal insertion sites for phages and plasmids. J Bacteriol 174, 7495-7499.

Churchward, G., Belin, D. \& Nagamine, Y. (1984). A pSC101derivative plasmid which shows no sequence homology to other commonly used cloning vectors. Gene 31, 165-171.

Clerget, M. (1991). Site-specific recombination promoted by a short DNA segment of plasmid R1 and by a homologous segment in the terminus region of the Escherichia coli chromosome. New Biol 3, 780-788.

Cohen, A., Bar-Nir, D., Goedeke, M. E. \& Parag, Y. (1985). The integrated and free states of Streptomyces griseus plasmid pSG1. Plasmid 13, 41-50.

Dorgai, L., Sloan, S. \& Weisberg, R. A. (1998). Recognition of core binding sites by bacteriophage integrases. J Mol Biol 277, 1059-1070.

Hanahan, D. (1983). Studies on transformation of Escherichia coli with plasmids. J Mol Biol 166, 557-580.

Madon, J., Moretti, P. \& Hutter, R. (1987). Site-specific integration and excision of pMEA100 in Nocardia mediterranei. Mol Gen Genet 209, 257-264.

Mazodier, P., Thompson, C. \& Boccard, F. (1990). The chromosomal integration site of the Streptomyces element pSAM2 overlaps a putative tRNA gene conserved among actinomycetes. Mol Gen Genet 222, 431-434.

Nakano, Y., Yoshida, Y., Yamashita, Y. \& Koga, T. (1995). Construction of a series of pACYC-derived plasmid vectors. Gene 162, 157-158.
Nunes-Düby, S. E., Kwon, H. J., Tirulalai, R. S., Ellenberg, T. \& Landy, A. (1998). Similarities and differences among 105 members of the Int family of site-specific recombinases. Nucleic Acids Res 26, 391-406.

Omer, C. A. \& Cohen, S. N. (1986). Structural analysis of plasmid and chromosomal loci involved in site-specific excision and integration of the SLP1 element of Streptomyces coelicolor. J Bacteriol 166, 999-1006.

Pena, C. E., Lee, M. H., Pedulla, M. L. \& Hatfull, G. F. (1997). Characterization of the mycobacteriophage L5 attachment site, attP. J Mol Biol 266, 76-92.

Pena, C. E. A., Kahlenberg, J. M. \& Hatfull, G. F. (1999). ProteinDNA complexes in mycobacteriophage L5 integrative recombination. J Bacteriol 181, 454-461.

Pena, C. E., Kahlenberg, J. M. \& Hatfull, G. F. (2000). Assembly and activation of site-specific recombination complexes. Proc Natl Acad Sci U S A 97, 7760-7765.

Pernodet, J. L., Simonet, J. M. \& Guerineau, M. (1984). Plasmids in different strains of Streptomyces ambofaciens: free and integrated form of plasmid pSAM2. Mol Gen Genet 198, 35-41.

Raynal, A., Tuphile, K., Gerbaud, C., Luther, T., Guerineau, M. \& Pernodet, J. L. (1998). Structure of the chromosomal insertion site for pSAM2: functional analysis in Escherichia coli. Mol Microbiol 28, 333-342.

Ross, W. \& Landy, A. (1982). Bacteriophage lambda Int protein recognizes two classes of sequence in the phage att site: characterization of arm-type sites. Proc Natl Acad Sci US A 79, 7724-7728.

Sambrook, J., Fritsch, E. F. \& Maniatis, T. (1989). Molecular Cloning: a Laboratory Manual. Cold Spring Harbor, NY: Cold Spring Harbor Laboratory.

Smith-Mungo, L., Chan, I. T. \& Landy, A. (1994). Structure of the P22 att site. Conservation and divergence in the lambda motif of recombinogenic complexes. J Biol Chem 269, 20798-20805.

Smokvina, T., Mazodier, P., Boccard, F., Thompson, C. J. \& Guerineau, M. (1990). Construction of a series of pSAM2-based integrative vectors for use in actinomycetes. Gene 94, 53-59.

Smokvina, T., Boccard, F., Pernodet, J. L., Friedmann, A. \& Guerineau, M. (1991). Functional analysis of the Streptomyces ambofaciens element pSAM2. Plasmid 25, 40-52.

Sosio, M., Madon, J. \& Hutter, R. (1989). Excision of pIJ408 from the chromosome of Streptomyces glaucescens and its transfer into Streptomyces lividans. Mol Gen Genet 218, 169-176.

Tirumalai, R. S., Kwon, H. J., Ellenberg, T. \& Landy, A. (1998). Recognition of core-type DNA sites by lambda integrase. J Mol Biol 279, 513-527.

Vrijbloed, J. W., Madon, J. \& Dijkhuizen, L. (1994). A plasmid from the methylotrophic actinomycete Amycolatopsis methanolica capable of site-specific integration. J Bacteriol 176, 7087-7090.

Yu, A. \& Haggard-Ljungquist, E. (1993). Characterization of the binding sites of two proteins involved in the bacteriophage P2 site-specific recombination system. J Bacteriol 175, 1239-1249.

Received 9 July 2001; revised 26 September 2001; accepted

27 September 2001. 